\title{
ESTIMATIVA DA VIDA ÚTIL E DOS PARÂMETROS DE CRESCIMENTO DE BACTÉRIAS ÁCIDO-LÁCTICAS EM FILÉS DE PEITO DE FRANGO RESFRIADOS EMBALADOS A VÁCUO E COM ATMOSFERA MODIFICADA
}

\author{
MARIA ELIZABETH DE PAULA CANÇADO MEZAROBA* \\ DANIEL ANGELO LONGHI** \\ ELIZANDRO VEDOVATTO*** \\ GLÁUCIA MARIA FALCÃO DE ARAGÃO****
}

\begin{abstract}
As bactérias ácido-lácticas (BAL) fazem parte da microflora natural e correspondem à principal população deteriorante de produtos cárneos embalados a vácuo e com atmosfera modificada (ATM), e seu crescimento é um dos fatores que definem a vida útil do produto. O objetivo deste trabalho foi quantificar a vida útil e os parâmetros do crescimento (duração da fase lag e velocidade máxima específica de crescimento) de BAL em filés de peito de frango resfriados embalados a vácuo e com ATM $\left(50 \%\right.$ de $\mathrm{CO}_{2}$ e $50 \%$ de $\mathrm{N}_{2}$ ) em diferentes temperaturas $\left(1{ }^{\circ} \mathrm{C}, 4{ }^{\circ} \mathrm{C}, 8{ }^{\circ} \mathrm{C}, 12{ }^{\circ} \mathrm{C}\right.$ e $20{ }^{\circ} \mathrm{C}$ ). Os parâmetros foram estimados com os modelos de Gompertz modificado, Baranyi e Logístico. Os modelos de Gompertz modificado e Baranyi apresentaram ajustes com melhores índices estatísticos ( $R^{2}$ e MSE e fatores bias e exatidão), sendo o modelo de Baranyi ligeiramente superior. Os modelos secundários Exponencial e Logarítmico foram utilizados para descrever a dependência dos parâmetros de crescimento em relação à temperatura, pois obtiveram melhores índices estatísticos. Os filés de peito de frango armazenados com ATM tiveram vida útil mais longa em comparação com os filés embalados a vácuo. Os modelos matemáticos estabelecidos neste estudo podem ser utilizados para predizer o crescimento das BAL em embalagem a vácuo e com ATM no intervalo de temperatura entre $1^{\circ} \mathrm{C}$ e $20^{\circ} \mathrm{C}$.
\end{abstract}

PALAVRAS-CHAVE: CARNE DE FRANGO; MICROBIOLOGIA PREDITIVA; PRESERVAÇÃO DE ALIMENTOS.

* Doutoranda em Engenharia de Alimentos, Departamento de Engenharia Química e Engenharia de Alimentos, Universidade Federal de Santa Catarina, Florianópolis - SC, Brasil (e-mail: mmezaroba@gmail.com).

** Doutorando em Engenharia de Alimentos, Departamento de Engenharia Química e Engenharia de Alimentos, Universidade Federal de Santa Catarina, Florianópolis - SC, Brasil (e-mail: ealdaniel@hotmail.com).

*** Mestrando em Engenharia de Alimentos, Departamento de Engenharia de Alimentos, Universidade Regional Integrada - Campus Erechim, Erechim - RS, Brasil (e-mail: vedovatto@auroraalimentos.com.br).

**** Professora doutora em Engenharia de Alimentos, Departamento de Engenharia Química e Engenharia de Alimentos, Universidade Federal de Santa Catarina, Florianópolis - SC, Brasil (e-mail: glaucia@enq.ufsc.br). 


\section{INTRODUÇÃO}

As carnes são um excelente substrato para o desenvolvimento de bactérias deteriorantes e patogênicas. A deterioração é fortemente determinada pelo crescimento de bactérias em sua superfície, uma vez que o tecido interno do músculo é considerado estéril até o momento do corte. Os tipos de microrganismos deteriorantes que se desenvolvem em carnes resfriadas são determinados pelas condições de estocagem (HOLLEY et al., 2004; GILL e GILL, 2005; IRKIN et al., 2011; CORTEZ-VEGA et al., 2012).

As bactérias ácido-lácticas (BAL) são principalmente mesófilas (com algumas linhagens termófilas), com temperatura ótima de crescimento numa faixa de $25^{\circ} \mathrm{C}$ a $40{ }^{\circ} \mathrm{C}$, e podem tolerar temperaturas mínimas entre $5{ }^{\circ} \mathrm{C}$ e $25^{\circ} \mathrm{C}$ e máximas entre $40^{\circ} \mathrm{C}$ e $50^{\circ} \mathrm{C}$. Esses microrganismos são ácido-tolerantes e podem crescer em valores de $\mathrm{pH}$ baixos, como 3,2 , e valores altos, como 9,6, e sobreviveriam naturalmente em pH entre 4,0 e 4,5 (FORSYTHE, 2002; RAMIREZ et al., 2011). A deterioração causada por essas bactérias é primordialmente devido à produção de metabólitos que causam mudanças indesejáveis na aparência, textura e flavor do alimento, produzindo odores e sabores desagradáveis, além de formar limo na superfície dos produtos (BORCH et al., 1996; SAMELIS et al., 2000; CAYRÉ et al., 2003; NYCHAS et al., 2008).

O tempo de vida útil é um atributo importante de todos os alimentos. Pode ser definido como o tempo que se passa desde a produção e a embalagem até o ponto em que o alimento se torna inaceitável para o consumo (FORSYTHE, 2002; SINGH e SINGH, 2005). Latou et al. (2014), em trabalho realizado em filé de peito de frango com tratamento em amostras controle (ar), ATM e vácuo, verificaram que as características organolépticas de sabor e odor ficaram com notas de aceitação abaixo do aceitável para contagens de BAL entre $10^{3}$ e $10^{4} \mathrm{UFC} / \mathrm{g}$. De acordo com os mesmos autores, existe correlação significativa entre a contagem de BAL e o odor das amostras.

A embalagem com atmosfera modificada (ATM) é reconhecida como uma das aplicações mais eficazes para a extensão da vida útil de produtos frescos e é amplamente utilizada pela indústria de carne. A eficácia da embalagem com ATM no prolongamento da vida útil de carnes é baseada na atividade antimicrobiana do $\mathrm{CO}_{2}$. O período de estocagem dos alimentos é consideravelmente prolongado pela modificação da atmosfera que circunda o produto e diminui a atividade dos microrganismos presentes (JAYAS e JEYAMKONDAN, 2002; KOUTSOUMANIS et al., 2008; DEGIRMENCIOGLU et al., 2012).

A microbiologia preditiva é uma ferramenta que pode ajudar a quantificar a qualidade e a segurança microbiológica dos alimentos através do uso de modelos matemáticos derivados de estudos quantitativos do crescimento microbiano (NAKASHIMA et al., 2000; LONGHI et al., 2013). Em microbiologia preditiva, os modelos primários descrevem a variação da concentração ou outra resposta microbiana com o tempo, e os modelos secundários descrevem como os parâmetros obtidos no modelo primário variam com as condições ambientais (WHITING e BUCHANAN, 1993).

O objetivo deste trabalho foi quantificar a vida útil e parâmetros de crescimento de BAL em filés de peito de frango resfriados embalados a vácuo e com ATM $\left(50 \%\right.$ de $\mathrm{CO}_{2}$ e $50 \%$ de $\mathrm{N}_{2}$ ) através do uso de diferentes modelos matemáticos em distintas temperaturas indicadas para comercialização $\left(1^{\circ} \mathrm{C}\right.$ e $\left.4{ }^{\circ} \mathrm{C}\right)$ e em temperaturas mais elevadas $\left(8^{\circ} \mathrm{C}, 12^{\circ} \mathrm{C}, 20^{\circ} \mathrm{C}\right)$.

\section{MATERIAL E MÉTODOS}

\subsection{AMOSTRAS}

As amostras de filé de peito de frango, sem pele, sem osso e pesando em torno de 400 
gramas (200 gramas cada metade), foram retiradas da linha de abate (todas pertencentes ao mesmo lote) de um frigorífico de aves localizado no estado de Santa Catarina, resfriadas a $0{ }^{\circ} \mathrm{C}$ e posteriormente embaladas a vácuo e em atmosfera modificada. As amostras não passaram por tratamentos para eliminação de microrganismos, sendo que a contaminação natural das amostras com BAL (com contagem inicial, em média, de $2 \times 10^{1} \mathrm{UFC} / \mathrm{g}$ ) foi considerada para a avaliação do crescimento microbiano.

\subsection{EMBALAGEM E ARMAZENAMENTO}

As amostras de filé de peito de frango foram acondicionadas em sacos plásticos e aplicaram-se os tratamentos de vácuo e ATM $\left(50 \%\right.$ de $\mathrm{CO}_{2}$ e $50 \%$ de $\left.\mathrm{N}_{2}\right)$. Em seguida, as amostras foram estocadas em condições de armazenamento isotérmico nas temperaturas de $1^{\circ} \mathrm{C}, 4^{\circ} \mathrm{C}, 8^{\circ} \mathrm{C}$, $12^{\circ} \mathrm{C} \mathrm{e} 20^{\circ} \mathrm{C}$ até que fosse observada a fase estacionária do crescimento microbiano. $\mathrm{O}$ registro de temperatura das amostras foi realizado a cada cinco minutos com auxílio de um datalogger (Akso, AK 285).

Os sacos plásticos utilizados como embalagem eram filmes multicamada coextrusados, com camada selante à base de polietileno (PELBD), camada externa à base de nylon e barreira a oxigênio (EVOH). Os sacos plásticos possuem barreira com baixa permeabilidade aos gases. Ao oxigênio, ela é menor que $3 \mathrm{~cm}^{3} / \mathrm{m}^{2}$.dia (a $23^{\circ} \mathrm{C}, 0 \% \mathrm{UR}$ ), e, ao vapor d'água, é menor que $6 \mathrm{~g} \mathrm{H}_{2} \mathrm{O} /$ $\mathrm{m}^{2}$.dia (a $38^{\circ} \mathrm{C}, 90 \%$ UR), com espessura de $125 \mu \mathrm{m}$ e as seguintes dimensões: $200 \mathrm{~mm}$ de largura x $260 \mathrm{~mm}$ de comprimento.

O equipamento utilizado para a embalagem foi a embaladora Microvac CV08, marca Selovac. O equipamento permite embalar a vácuo e com ATM, pois o funcionamento do sistema é por substituição mecânica do ar (técnica do vácuo compensado). O cilindro com a mistura gasosa para a embalagem com ATM ( $50 \%$ de $\mathrm{CO}_{2}$ e $50 \%$ de $\mathrm{N}_{2}$ ) foi cedido pela empresa Linde Gases Ltda.

\subsection{ANÁLISES MICROBIOLÓGICAS}

Amostras de filé de peito de frango foram retiradas do armazenamento isotérmico periodicamente. Amostragens pesando 25 gramas (balança digital Marte, modelo AS 5000) foram coletadas em câmara de fluxo laminar (Valiclean, modelo CL II) utilizando-se saco plástico estéril e homogeneizadas em solução salina peptonada $(0,85 \% \mathrm{NaCl} ; 0,1 \%$ peptona) durante um minuto em Stomacher (Intersciense, modelo Bag Mixer). Em seguida, as diluições adequadas foram preparadas em duplicata e semeadas em placas de Petri descartáveis e estéreis em meio de cultura MRS (Man, Rogosa e Sharpe, marca Merck) em dupla camada para contagem de bactérias lácticas. As placas semeadas foram incubadas em incubadora biológica (Biopar modelo $150 \mathrm{~L}$ ) à temperatura de incubação de $30^{\circ} \mathrm{C} \pm 1{ }^{\circ} \mathrm{C}$, com tempo de incubação de 48 horas. Após esse período, realizou-se a contagem de unidades formadoras de colônia (UFC) presentes nas placas. Para tanto, utilizou-se o contador de colônias tipo Quebec. O resultado foi expresso em unidades formadoras de colônia por grama de amostra (UFC/g).

\subsection{MODELAGEM MATEMÁTICA}

Os modelos matemáticos primários de Gompertz modificado e Logístico (ZWIETERING et al., 1990), Equações (1) e (2), respectivamente, e Baranyi (BARANYI e ROBERTS, 1994), Equações (3) e (4), foram selecionados e utilizados para descrever a variação da concentração microbiana com o tempo. Esses modelos são apresentados na tabela 1. 
TABELA 1 - EQUAÇÕES DOS MODELOS PRIMÁRIOS DE CRESCIMENTO

\begin{tabular}{ccc}
\hline Modelo & Equação & \\
\hline Gompertz modificado & $y(t)=y_{0}+A \exp \{-\exp [(\exp (1) \mu(\lambda-t) / A)+1]\}$ & $(1)$ \\
\hline Logístico & $y(t)=y_{0}+\{A /[1+\exp [(4 \mu(\lambda-t) / A)+1]\}$ & $(3)$ \\
\hline Baranyi & $y(t)=y_{0}+\mu F(t)-\ln \left\{1+[\exp (\mu F(t))-1] /\left[\exp \left(y_{\max }-y_{0}\right]\right\}\right.$ & $(4)$ \\
\cline { 2 - 3 }
\end{tabular}

Onde: $y(t)=$ logaritmo neperiano da concentração microbiana (In UFC/g) no tempo $t$ (dias); $y_{0}$ e $y_{\max }=$ logaritmo neperiano da concentração microbiana inicial e máxima (In UFC/g), respectivamente; $A$ = aumento logarítmico da população microbiana; $\mu$ $=$ velocidade específica máxima de crescimento $\left(\right.$ dias $\left.^{-1}\right) ; \lambda=$ duração da fase lag (dias); $F(t)=$ função do modelo de Baranyi e Roberts relacionada com o estado fisiológico das células.

A temperatura influencia os parâmetros de crescimento $\mu, \lambda$ e $A$ obtidos dos modelos primários, sendo que os modelos secundários Linear, Equação (5), Raiz Quadrada (RATKOWSKY et al., 1982), Equação (6), Logarítmico, Equação (7), Exponencial e Equação (8) foram utilizados para descrever a dependência dos parâmetros em relação à temperatura. A tabela 2 apresenta as equações dos modelos secundários citados. O tempo de vida útil dos filés de peito de frango foi definido neste trabalho como o tempo em que a população microbiana atingiu a concentração de $10^{3}$ UFC/g, baseado em Latou et al. (2014). Toda a modelagem matemática foi realizada com o software Matlab 2010a (Natick, USA).

\section{TABELA 2 - EQUAÇÕES DOS MODELOS SECUNDÁRIOS}

\begin{tabular}{clc}
\hline Modelo & Equação & \\
\hline Linear & $p=a+c T$ & $(5)$ \\
\hline Raiz Quadrada & $p=b\left(T-T_{\min }\right)$ & $(6)$ \\
\hline Logarítmico & $\ln (p)=d T+e$ & $(7)$ \\
\hline \hline Exponencial & $p=f \exp (g T)$ & $(8)$ \\
\hline \hline
\end{tabular}

Onde: $p=$ parâmetro de interesse do modelo primário $(\mu, \lambda$ ou A $) ; T=$ temperatura $\left({ }^{\circ} \mathrm{C}\right) ; T_{\min }=$ temperatura mínima teórica para o crescimento microbiano; $a, b, c, d, e, f$ e $g$ = parâmetros empíricos.

\section{ANÁLISES ESTATÍSTICAS}

Os índices estatísticos coeficiente de correlação $\left(R^{2}\right)$ e erro médio quadrático (MSE), bem como os fatores bias e exatidão (ROSS, 1996), foram utilizados para avaliar a qualidade de ajuste dos modelos matemáticos.

\section{RESULTADOS E DISCUSSÃO}

Os valores dos índices estatísticos $\mathrm{R}^{2}$ e MSE, bem como os fatores bias e exatidão resultantes dos ajustes dos modelos de Gompertz modificado, Logístico e Baranyi aos dados de crescimento das BAL nas diferentes temperaturas $\left(1^{\circ} \mathrm{C}, 4^{\circ} \mathrm{C}, 8^{\circ} \mathrm{C}, 12{ }^{\circ} \mathrm{C}\right.$ e $\left.20^{\circ} \mathrm{C}\right)$ para embalagem com ATM e a vácuo, são apresentados nas tabelas 3 e 4 respectivamente.

Ao analisar os dados delas é possível verificar através dos índices estatísticos (principalmente pelo fator de exatidão) que os modelos de Gompertz modificado e Baranyi apresentaram melhores capacidades de ajuste aos dados isotérmicos (para várias condições) do que o modelo Logístico, para ambos os tipos de embalagem (a vácuo e com ATM).

Por terem apresentado os melhores índices estatísticos, os ajustes dos modelos de Gompertz modificado e Baranyi aos dados experimentais do crescimento de BAL em filé de peito de frango resfriado para a embalagem com ATM são apresentados nas figuras 1 e 2 , respectivamente, e, para a embalagem a vácuo, nas figuras 3 e 4, respectivamente. 
TABELA 3 - VALORES DOS ÍNDICES ESTATÍSTICOS DOS AJUSTES DOS MODELOS DE GOMPERTZ MODIFICADO, LOGÍSTICO E BARANYI AOS DADOS DE CRESCIMENTO DE BAL OBTIDOS A $1^{\circ} \mathrm{C}, 4^{\circ} \mathrm{C}, 8^{\circ} \mathrm{C}, 12^{\circ} \mathrm{C}$ E $20^{\circ} \mathrm{C}$ PARA A EMBALAGEM COM ATM

\begin{tabular}{|c|c|c|c|c|c|}
\hline $\mathrm{T}\left({ }^{\circ} \mathrm{C}\right)$ & Modelo & MSE & Bias & Exatidão & $\mathrm{R}^{2}$ \\
\hline \multirow{3}{*}{1} & Gompertz modificado & 2,51 & 0,98 & 1,15 & 0,83 \\
\hline & Logístico & 1,79 & 1,08 & 1,35 & 0,82 \\
\hline & Baranyi & 1,92 & 1,00 & 1,14 & 0,83 \\
\hline \multirow{3}{*}{4} & Gompertz modificado & 0,79 & 1,05 & 1,13 & 0,97 \\
\hline & Logístico & 1,03 & 0,76 & 1,51 & 0,95 \\
\hline & Baranyi & 2,20 & 1,10 & 1,23 & 0,96 \\
\hline \multirow{3}{*}{8} & Gompertz modificado & 0,74 & 1,00 & 1,06 & 0,97 \\
\hline & Logístico & 0,76 & 1,03 & 1,25 & 0,97 \\
\hline & Baranyi & 0,68 & 1,06 & 1,06 & 0,98 \\
\hline \multirow{3}{*}{12} & Gompertz modificado & 2,83 & 1,03 & 1,12 & 0,86 \\
\hline & Logístico & 3,19 & 1,06 & 1,23 & 0,86 \\
\hline & Baranyi & 2,38 & 1,03 & 1,11 & 0,85 \\
\hline \multirow{3}{*}{20} & Gompertz modificado & 1,09 & 1,01 & 1,10 & 0,96 \\
\hline & Logístico & 2,12 & 0,96 & 1,13 & 0,93 \\
\hline & Baranyi & 1,96 & 0,98 & 1,10 & 0,92 \\
\hline
\end{tabular}

TABELA 4 - VALORES DOS ÍNDICES ESTATÍSTICOS DOS AJUSTES DOS MODELOS DE GOMPERTZ MODIFICADO, LOGÍSTICO E BARANYI AOS DADOS DE CRESCIMENTO DE BAL OBTIDOS A $1^{\circ} \mathrm{C}, 4^{\circ} \mathrm{C}, 8^{\circ} \mathrm{C}, 12^{\circ} \mathrm{C}$ E $20^{\circ} \mathrm{C}$ PARA A EMBALAGEM A VÁCUO

\begin{tabular}{|c|c|c|c|c|c|}
\hline $\mathrm{T}\left({ }^{\circ} \mathrm{C}\right)$ & Modelo & MSE & Bias & Exatidão & $\mathrm{R}^{2}$ \\
\hline \multirow{3}{*}{1} & Gompertz modificado & 1,66 & 1,02 & 1,13 & 0,88 \\
\hline & Logístico & 1,11 & 1,00 & 1,29 & 0,87 \\
\hline & Baranyi & 1,55 & 1,00 & 1,13 & 0,88 \\
\hline \multirow{3}{*}{4} & Gompertz modificado & 5,04 & 1,08 & 1,21 & 0,97 \\
\hline & Logístico & 1,23 & 1,00 & 1,04 & 0,97 \\
\hline & Baranyi & 2,93 & 0,98 & 1,19 & 0,95 \\
\hline \multirow{3}{*}{8} & Gompertz modificado & 1,34 & 1,01 & 1,10 & 0,95 \\
\hline & Logístico & 1,21 & 0,97 & 1,19 & 0,95 \\
\hline & Baranyi & 1,53 & 1,01 & 1,10 & 0,95 \\
\hline \multirow{3}{*}{12} & Gompertz modificado & 2,43 & 1,03 & 1,11 & 0,87 \\
\hline & Logístico & 2,82 & 1,13 & 1,33 & 0,87 \\
\hline & Baranyi & 2,58 & 1,00 & 1,11 & 0,90 \\
\hline \multirow{3}{*}{20} & Gompertz modificado & 1,13 & 1,00 & 1,09 & 0,96 \\
\hline & Logístico & 1,66 & 1,00 & 1,07 & 0,91 \\
\hline & Baranyi & 2,47 & 0,98 & 1,10 & 0,89 \\
\hline
\end{tabular}




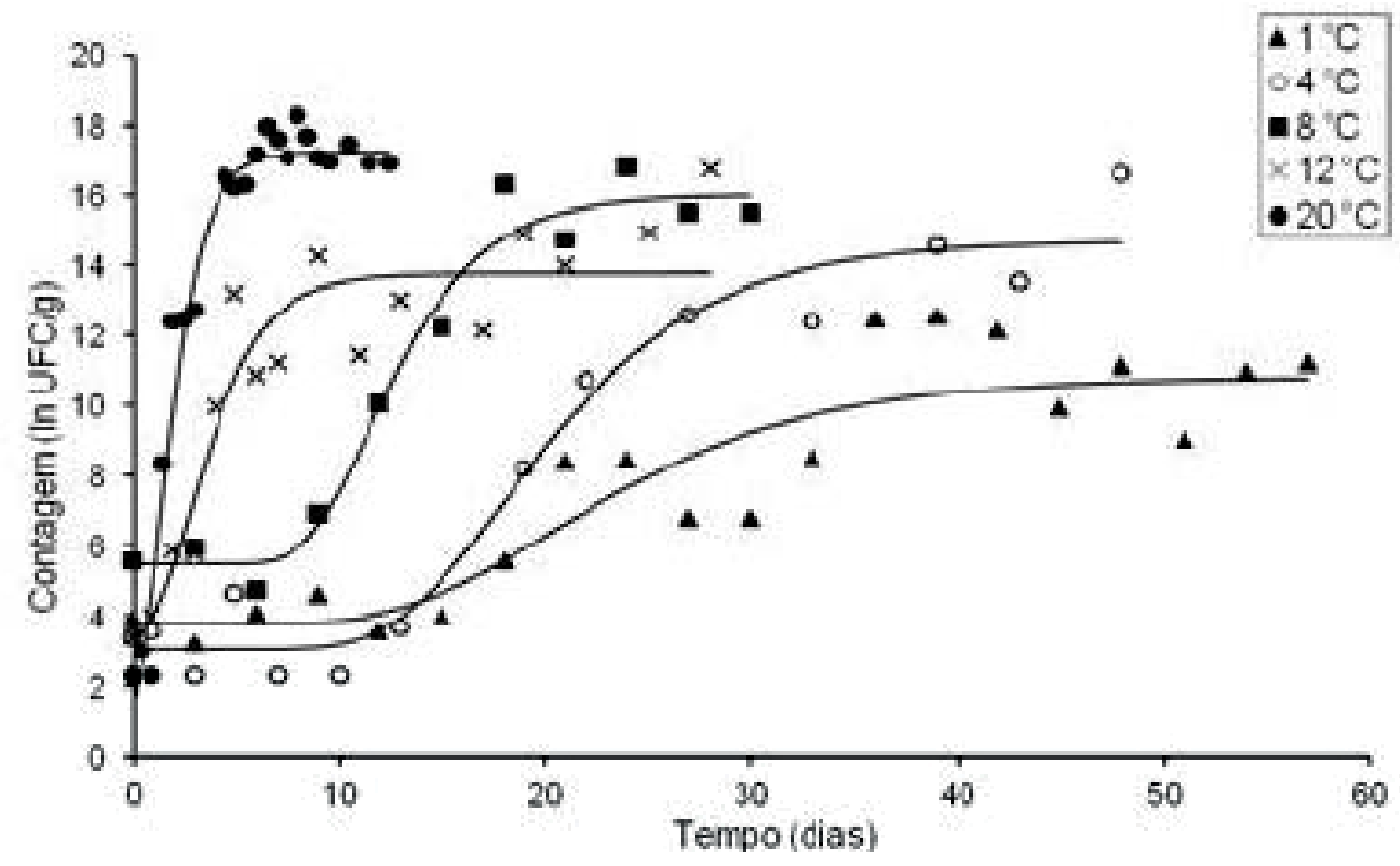

FIGURA 1 - AJUSTE DO MODELO DE GOMPERTZ MODIFICADO (LINHA CONTÍNUA) AOS DADOS EXPERIMENTAIS DO CRESCIMENTO DE BAL (SÍMBOLOS) EM FILÉS DE PEITO DE FRANGO EMBALADOS COM ATM NAS TEMPERATURAS DE $1^{\circ} \mathrm{C}, 4^{\circ} \mathrm{C}, 8^{\circ} \mathrm{C}$, $12{ }^{\circ} \mathrm{C}$ E $20^{\circ} \mathrm{C}$

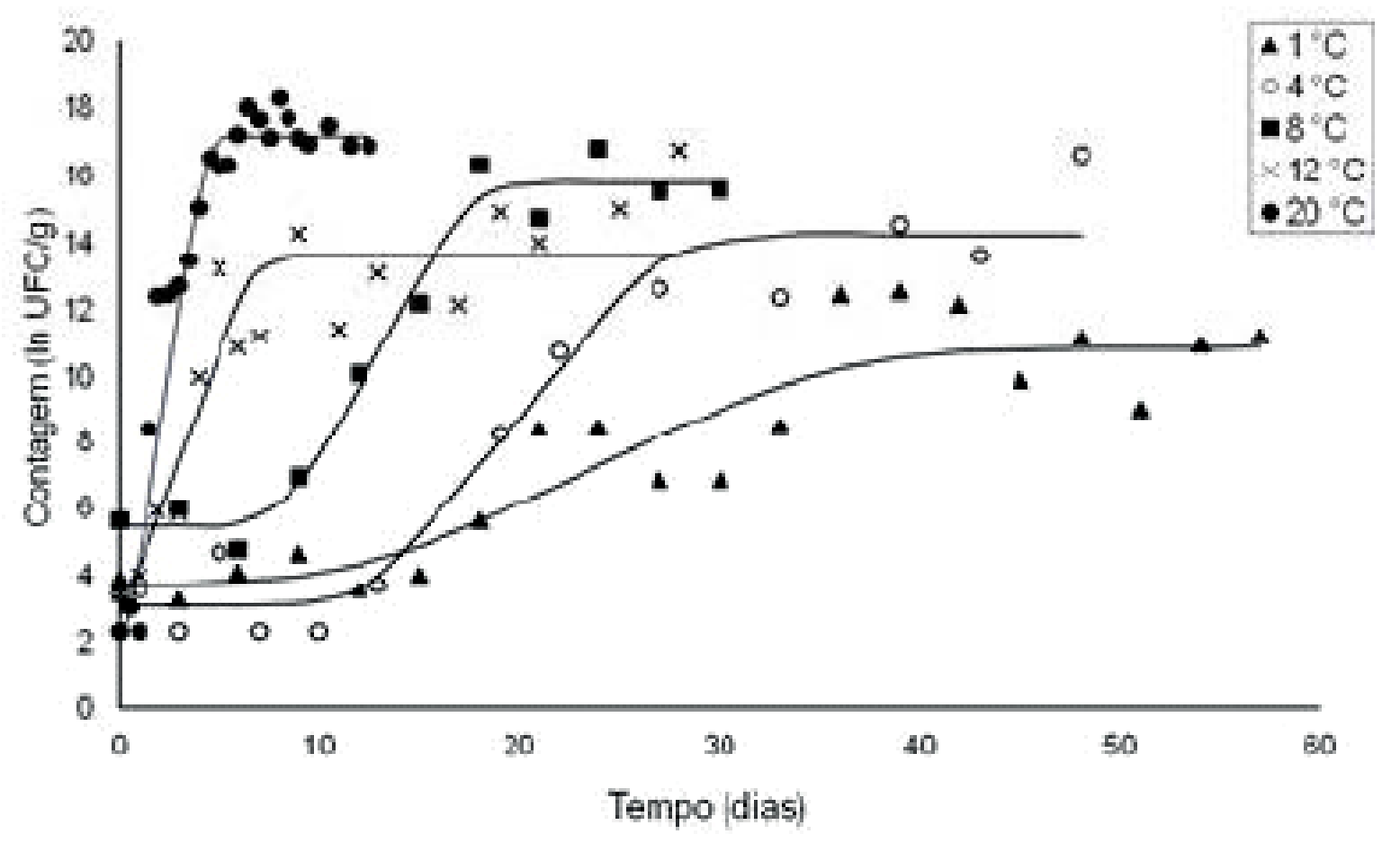

FIGURA 2 - AJUSTE DO MODELO DE BARANYI (LINHA CONTÍNUA) AOS DADOS EXPERIMENTAIS DO CRESCIMENTO DE BAL (SÍMBOLOS) EM FILÉS DE PEITO DE FRANGO EMBALADOS COM ATM NAS TEMPERATURAS DE $1^{\circ} \mathrm{C}, 4^{\circ} \mathrm{C}, 8^{\circ} \mathrm{C}, 12^{\circ} \mathrm{C}$ E 20 


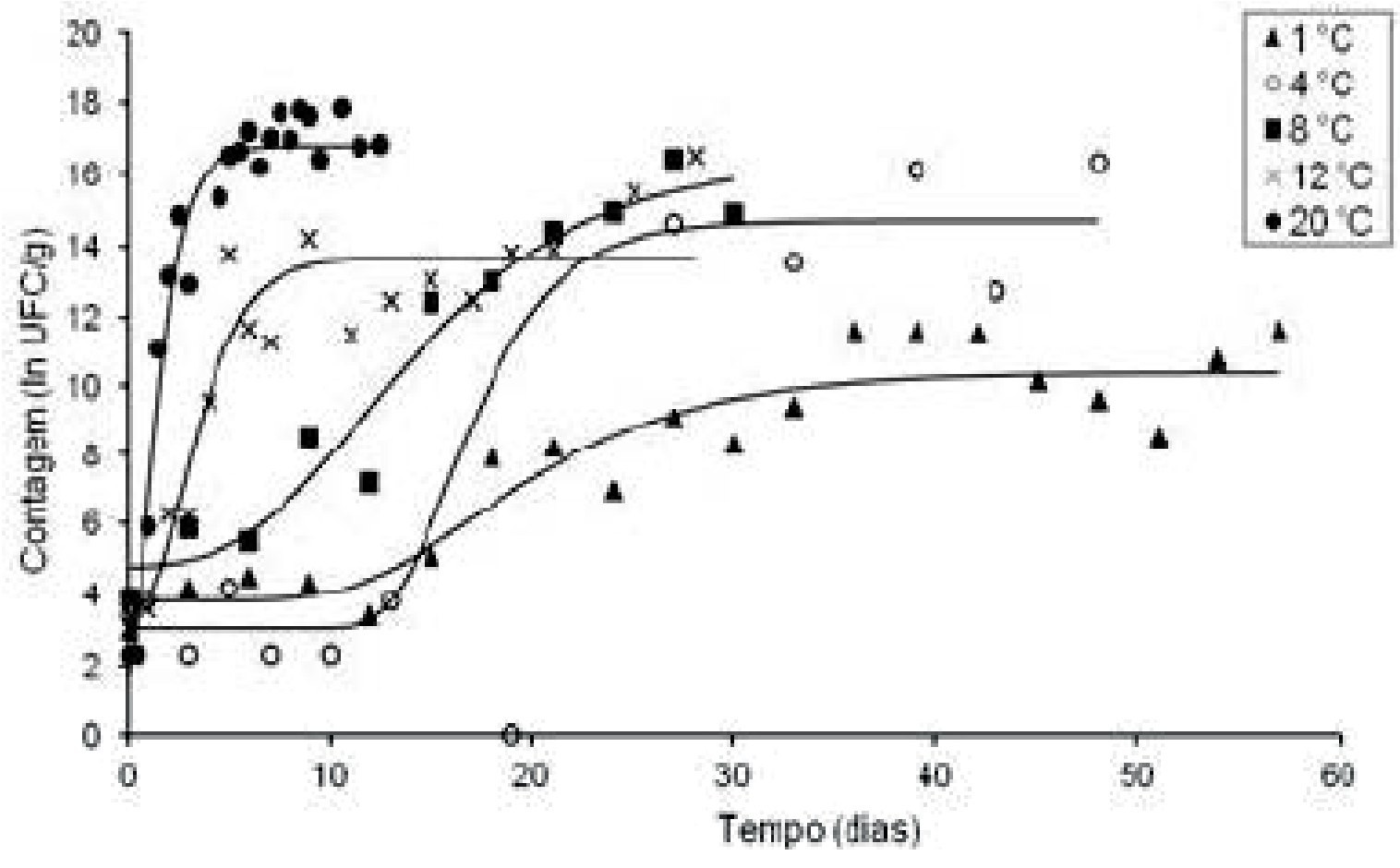

FIGURA 3 - AJUSTE DO MODELO DE GOMPERTZ MODIFICADO (LINHA CONTÍNUA) AOS DADOS EXPERIMENTAIS DO CRESCIMENTO DE BAL (SÍMBOLOS) EM FILÉS DE PEITO DE FRANGO EMBALADOS A VÁCUO NAS TEMPERATURAS DE $1^{\circ} \mathrm{C}, 4^{\circ} \mathrm{C}, 8^{\circ} \mathrm{C}, 12^{\circ} \mathrm{C}$ E $20^{\circ} \mathrm{C}$

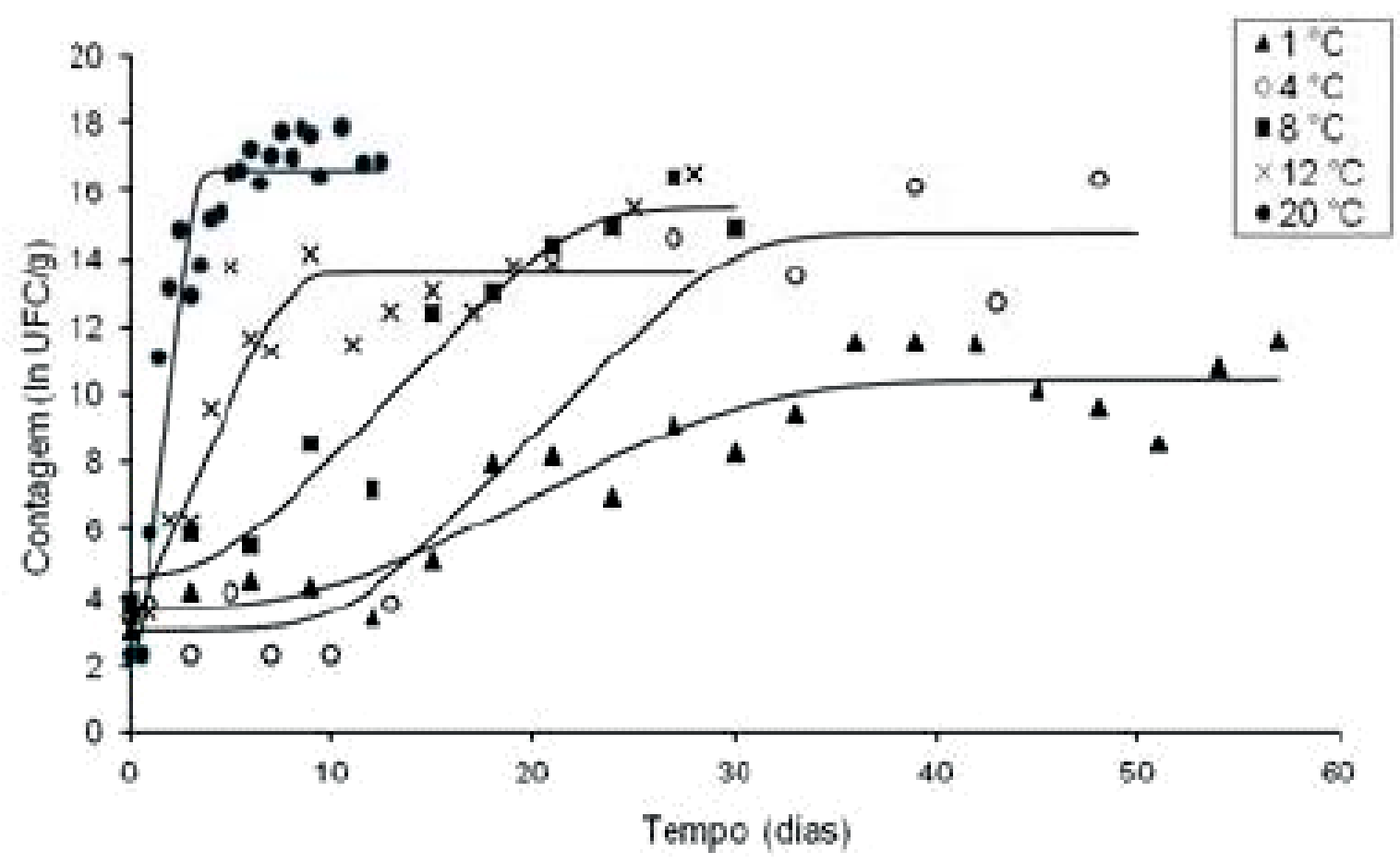

FIGURA 4 - AJUSTE DO MODELO DE BARANYI (LINHA CONTÍNUA) AOS DADOS EXPERIMENTAIS DO CRESCIMENTO DE BAL (SÍMBOLOS) EM FILÉS DE PEITO DE FRANGO EMBALADOS A VÁCUO NAS TEMPERATURAS DE 1 ${ }^{\circ} \mathrm{C}, 4^{\circ} \mathrm{C}, 8^{\circ} \mathrm{C}, 12{ }^{\circ} \mathrm{C} \mathrm{E} 20^{\circ} \mathrm{C}$ 
Ao analisar as figuras 1, 2, 3 e 4, é possível verificar que, com a diminuição da temperatura, há o aumento na fase lag e a diminuição da velocidade específica máxima de crescimento e da população máxima atingida pelas BAL (exceto para a curva de $12{ }^{\circ} \mathrm{C}$, que apresentou menor valor para a população máxima atingida do que a curva de $8{ }^{\circ} \mathrm{C}$, o que pode estar relacionado com a contagem inicial). Consequentemente, o armazenamento dos peitos de frango em temperaturas mais baixas aumentou a sua vida útil, tanto daqueles embalados a vácuo como daqueles embalados com ATM.

Os valores dos parâmetros de crescimento $\mu, \lambda$ e $y_{\max }$, bem como os valores da vida útil de peitos de frango embalados a vácuo e com ATM em cada temperatura, são apresentados nas tabelas 5 e 6 respectivamente. Ao analisá-las, é possível verificar que o modelo de Baranyi apresenta, em geral, valores maiores para os parâmetros $\mu, \lambda$ e vida útil em relação ao modelo de Gompertz modificado.

\section{TABELA 5 - PARÂMETROS DE CRESCIMENTO DAS BAL EM FILÉS DE PEITO DE FRANGO EMBALADOS COM ATM}

\begin{tabular}{|c|c|c|c|c|c|}
\hline Modelo & $\mathrm{T}\left({ }^{\circ} \mathrm{C}\right)$ & $\mu\left(\right.$ dias $\left.^{-1}\right)$ & $\lambda$ (dias) & $\mathbf{y}_{\max }$ & Vida útil (dias) \\
\hline \multirow{5}{*}{ Gompertz modificado } & 1 & 0,338 & 13,15 & 11,67 & 20,55 \\
\hline & 4 & 1,703 & 16,58 & 12,83 & 22,22 \\
\hline & 8 & 1,249 & 8,47 & 15,95 & 9,40 \\
\hline & 12 & 2,294 & 1,26 & 14,20 & 2,39 \\
\hline & 20 & 5,305 & 0,55 & 17,72 & 1,32 \\
\hline \multirow{5}{*}{ Baranyi } & 1 & 0,484 & 20,42 & 11,06 & 25,45 \\
\hline & 4 & 0,750 & 13,19 & 13,51 & 18,43 \\
\hline & 8 & 1,165 & 8,49 & 15,79 & 9,45 \\
\hline & 12 & 3,893 & 2,54 & 12,95 & 3,19 \\
\hline & 20 & 9,018 & 1,10 & 16,52 & 1,55 \\
\hline
\end{tabular}

TABELA 6 - PARÂMETROS DE CRESCIMENTO DAS BAL EM FILÉS DE PEITO DE FRANGO EMBALADOS A VÁCUO

\begin{tabular}{|c|c|c|c|c|c|}
\hline Modelo & $\mathrm{T}\left({ }^{\circ} \mathrm{C}\right)$ & $\mu\left(\right.$ dias $\left.^{-1}\right)$ & $\lambda$ (dias) & $y_{\max }$ & Vida útil (dias) \\
\hline \multirow{5}{*}{$\begin{array}{c}\text { Gompertz } \\
\text { modificado }\end{array}$} & 1 & 0,278 & 5,10 & 12,11 & 26,39 \\
\hline & 4 & 3,985 & 18,90 & 13,98 & 30,63 \\
\hline & 8 & 0,641 & 3,18 & 17,08 & 6,52 \\
\hline & 12 & 2,630 & 1,38 & 12,84 & 2,76 \\
\hline & 20 & 6,183 & 0,34 & 16,51 & 1,12 \\
\hline \multirow{5}{*}{ Baranyi } & 1 & 0,293 & 10,36 & 11,98 & 20,93 \\
\hline & 4 & 0,459 & 6,61 & 16,39 & 16,83 \\
\hline & 8 & 0,643 & 4,71 & 14,99 & 8,13 \\
\hline & 12 & 2,463 & 1,23 & 12,78 & 2,71 \\
\hline & 20 & 7,155 & 0,42 & 16,38 & 1,10 \\
\hline
\end{tabular}


A vida útil dos filés de peito de frango calculada por ambos os modelos diminuiu drasticamente com o aumento da temperatura. Ao analisar as tabelas 5 e 6 , é possível verificar que a vida útil dos filés de peito de frango diminuiu de mais de 20 dias a $1{ }^{\circ} \mathrm{C}$ para menos de dez dias a $8{ }^{\circ} \mathrm{C}$ e menos de 1,6 dia a $20^{\circ} \mathrm{C}$. É possível verificar também que, em geral, a vida útil foi maior para os filés de peito de frango embalados com ATM do que para aqueles embalados a vácuo. A diferença de vida útil calculada nas diferentes embalagens, porém, não é muito grande.

Os modelos secundários Raiz Quadrada, Linear, Logarítmico e Exponencial foram ajustados aos dados dos parâmetros $\mu, \lambda$ e vida útil dos modelos de Gompertz modificado e Baranyi. Os valores resultantes de $\mathrm{R}^{2}$ do ajuste dos modelos aos parâmetros são apresentados nas tabelas 7 e 8 respectivamente. Os modelos que apresentaram os melhores ajustes a cada parâmetro (medido através do $\mathrm{R}^{2}$ ) foram os selecionados.

TABELA 7 - VALORES DE R² OBTIDOS DO AJUSTE DOS MODELOS LINEAR, RAIZ QUADRADA, LOGARÍTMICO E EXPONENCIAL AOS DADOS DOS PARÂMETROS $\Lambda$, M E VIDA ÚTIL OBTIDOS PELOS MODELOS DE GOMPERTZ MODIFICADO E BARANYI PARA OS DADOS DE FILÉS DE PEITO DE FRANGO EMBALADOS COM ATM

\begin{tabular}{ccccc}
\hline Modelo primário & Modelo secundário & $\mu$ & $\lambda$ & Vida útil \\
\hline & Linear & 0,850 & 0,788 & 0,813 \\
\hline & Raiz Quadrada & 0,887 & 0,894 & 0,926 \\
\cline { 2 - 5 } Gompertz modificado & Logarítmico & 0,798 & 0,895 & 0,928 \\
\hline & Exponencial & 0,950 & 0,755 & 0,784 \\
\hline & Linear & 0,915 & 0,857 & 0,850 \\
\hline & Raiz Quadrada & 0,915 & 0,916 & 0,936 \\
\hline \hline & Logarítmico & 0,970 & 0,969 & 0,966 \\
\hline
\end{tabular}

TABELA 8 - VALORES DE R² OBTIDOS DO AJUSTE DOS MODELOS LINEAR, RAIZ QUADRADA, LOGARÍTMICO E EXPONENCIAL AOS DADOS DOS PARÂMETROS $\Lambda$, M E VIDA ÚTIL OBTIDOS PELOS MODELOS DE GOMPERTZ MODIFICADO E BARANYI PARA OS DADOS DE FILÉS DE PEITO DE FRANGO EMBALADOS A VÁCUO

\begin{tabular}{ccccc}
\hline Modelo primário & Modelo secundário & $\mu$ & $\lambda$ & Vida útil \\
\hline \hline \multirow{2}{*}{ Gompertz modificado } & Linear & 0,537 & 0,340 & 0,718 \\
\cline { 2 - 5 } & Raiz Quadrada & 0,537 & 0,803 & 0,931 \\
\cline { 2 - 5 } & Logarítmico & 0,477 & 0,831 & 0,940 \\
\hline \hline & Exponencial & 0,611 & 0,290 & 0,848 \\
\hline \hline & Raiz Quadrada & 0,881 & 0,869 & 0,863 \\
\hline \hline & Logarítmico & 0,881 & 0,880 & 0,913 \\
\hline \hline
\end{tabular}


Ao analisar as tabelas 7 e 8, é possível verificar que o modelo Exponencial foi o modelo secundário que melhor se ajustou aos dados dos parâmetro $\mu$ obtidos por ambos os modelos (de Gompertz modificado e Baranyi) para os dados de filés de peito de frango embalados em ambas as condições (com ATM e a vácuo). O modelo Exponencial também foi o modelo que melhor se ajustou aos dados do parâmetro $\lambda$ e vida útil obtidos pelo modelo de Baranyi para os dados de filés de peito de frango embalados em ambas as condições (com ATM e a vácuo). As exceções foram os parâmetros $\lambda$ e vida útil obtidos pelo modelo de Gompertz modificado, em que modelo Logarítmico melhor se ajustou aos dados.

Os modelos secundários que melhor se ajustaram aos dados dos parâmetros $\mu, \lambda$ e vida útil obtidos por ambos os modelos (de Gompertz modificado e Baranyi) para os dados de filés de peito de frango embalados em ambas as condições (com ATM e a vácuo) são apresentados nas figuras 5 e 6 , e as equações dos modelos com seus parâmetros ajustados são apresentadas na tabela 9 . Ao analisar as figuras 5 e 6 , é possível verificar de forma qualitativa que todos os modelos apresentaram bons ajustes aos dados experimentais, sendo que os modelos secundários apresentados na tabela 9 podem ser usados para predizer os parâmetros de crescimento das BAL nas condições estudadas e em condições que não extrapolem a temperatura no intervalo entre 1 ${ }^{\circ} \mathrm{C}$ e $20^{\circ} \mathrm{C}$.

\section{TABELA 9 - EQUAÇÕES DOS MODELOS SECUNDÁRIOS AJUSTADOS AOS DADOS DOS PARÂMETROS $\Lambda$, M E VIDA ÚTIL OBTIDOS PELOS MODELOS DE GOMPERTZ MODIFICADO E BARANYI PARA OS DADOS DE FILÉS DE PEITO DE FRANGO EMBALADOS A VÁCUO E COM ATM}

\begin{tabular}{|c|c|c|}
\hline Modelo & Embalagem & Equações \\
\hline \multirow{6}{*}{ Gompertz modificado } & \multirow{3}{*}{ ATM } & $\mu_{\mathrm{atm}}=0,669 \exp (0,103 \mathrm{~T})$ \\
\hline & & $\ln \left(\lambda_{\mathrm{atm}}\right)=-0,194 \mathrm{~T}+3,177$ \\
\hline & & In $\left(\right.$ Vida útil $\left.{ }_{\text {atm }}\right)=-0,165 T+3,386$ \\
\hline & \multirow{3}{*}{ Vácuo } & $\mu_{\mathrm{vac}}=0,997 \exp (0,090 \mathrm{~T})$ \\
\hline & & $\ln \left(\lambda_{\text {vac }}\right)=-0,181 T+2,621$ \\
\hline & & In $\left(\right.$ Vida útil $\left.{ }_{\text {vac }}\right)=-0,186 \mathrm{~T}+3,612$ \\
\hline \multirow{6}{*}{ Baranyi } & \multirow{3}{*}{ ATM } & $\mu_{\mathrm{atm}}=0,597 \exp (0,136 \mathrm{~T})$ \\
\hline & & $\lambda_{\mathrm{atm}}=23,93 \exp (-0,150 \mathrm{~T})$ \\
\hline & & Vida útil $\left.\right|_{\mathrm{atm}}=30,52 \exp (-0,150 \mathrm{~T})$ \\
\hline & \multirow{3}{*}{ Vácuo } & $\mu_{\mathrm{vac}}=0,303 \exp (0,158 \mathrm{~T})$ \\
\hline & & $\lambda_{\mathrm{vac}}=12,08 \exp (-0,146 \mathrm{~T})$ \\
\hline & & Vida útil $_{\mathrm{vac}}=25,49 \exp (-0,143 \mathrm{~T})$ \\
\hline
\end{tabular}



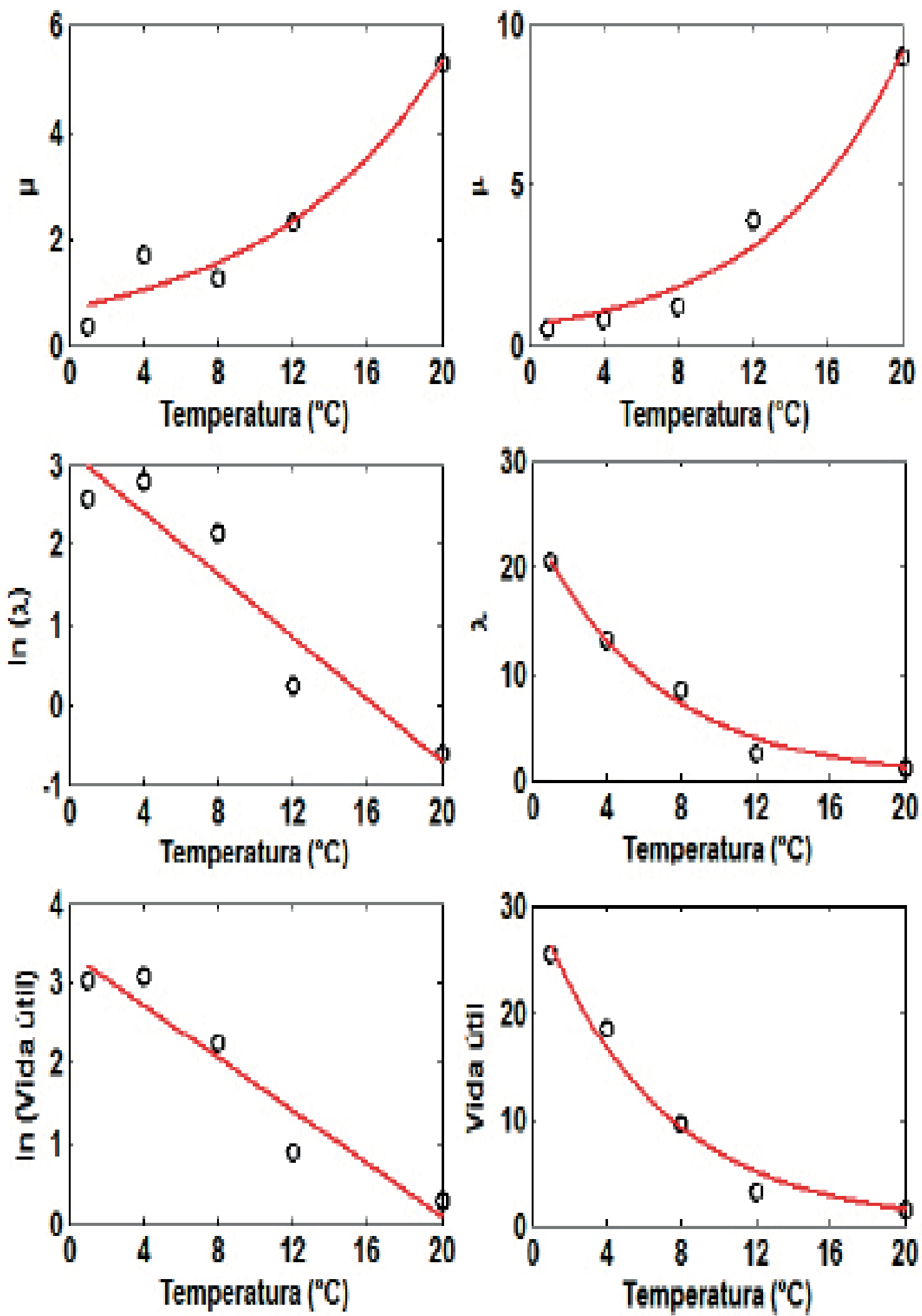

FIGURA 5 - AJUSTE DOS MODELOS SECUNDÁRIOS AOS DADOS DOS PARÂMETROS

$\Lambda, M$ E VIDA ÚTIL OBTIDOS PELOS MODELOS DE GOMPERTZ MODIFICADO (FIGURAS À ESQUERDA) E BARANYI (FIGURAS À DIREITA) PARA OS DADOS DE FILÉS DE PEITO DE FRANGO EMBALADOS COM ATM 

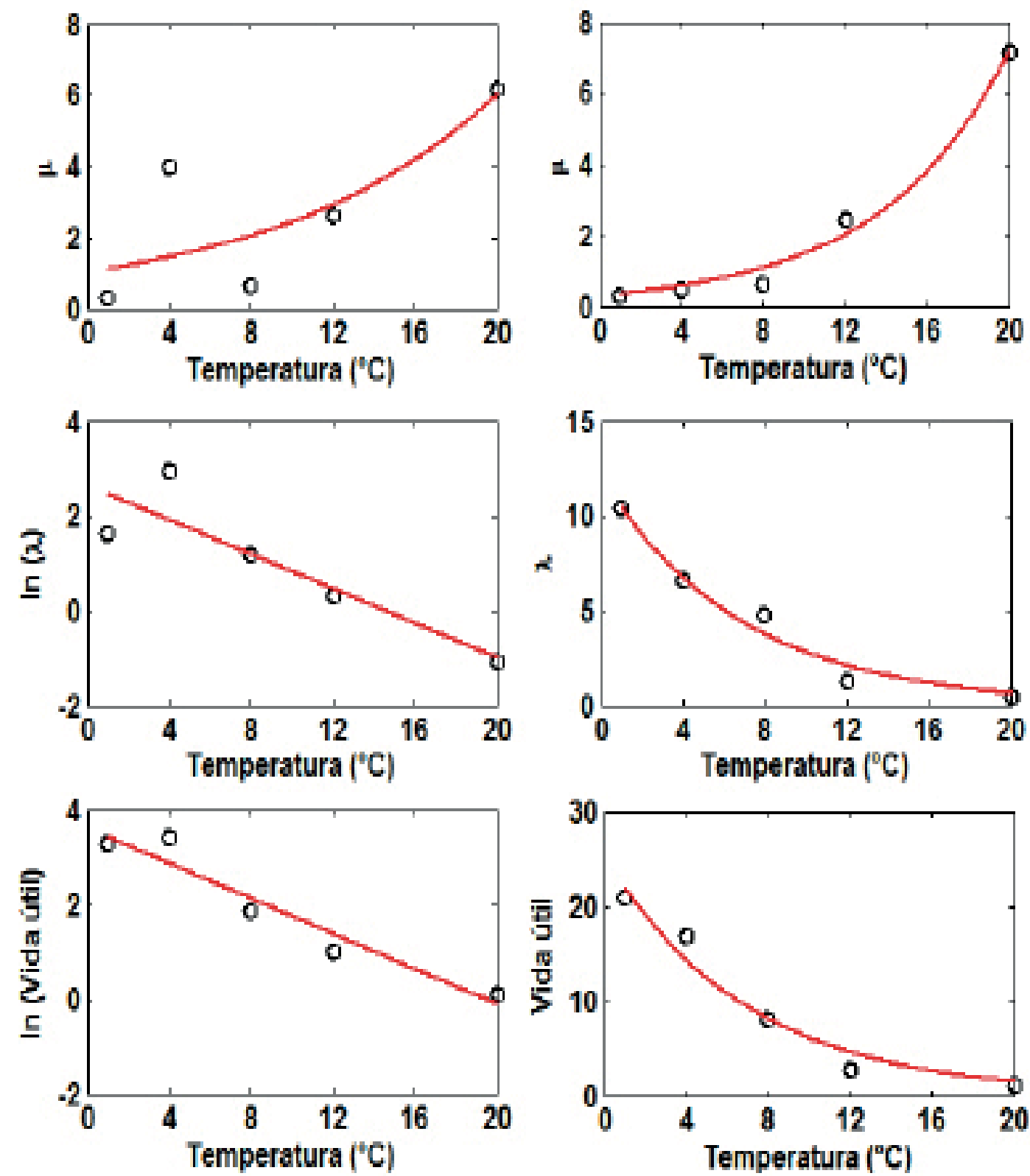

FIGURA 6 - AJUSTE DOS MODELOS SECUNDÁRIOS AOS DADOS DOS PARÂMETROS $\Lambda$, M E VIDA ÚTIL OBTIDOS PELOS MODELOS DE GOMPERTZ MODIFICADO (FIGURAS À ESQUERDA) E BARANYI (FIGURAS À DIREITA) PARA OS DADOS DE FILÉS DE PEITO DE FRANGO EMBALADOS A VÁCUO

\section{CONCLUSÃO}

Os modelos primários de Gompertz modificado e de Baranyi apresentaram ajustes com melhores índices estatísticos em relação ao modelo Logístico, e os modelos secundários Exponencial e Logarítmico obtiveram os melhores ajustes aos dados dos parâmetros $\mu, \lambda$ e vida útil. Os filés de frango armazenados com ATM tiveram maior vida útil em comparação com os filés embalados a vácuo. A vida útil dos filés de peito de frango, que era maior que 20 dias a $1^{\circ} \mathrm{C}$, diminuiu para menos de dez dias a $8^{\circ} \mathrm{C}$ e menos de 1,6 dia a $20^{\circ} \mathrm{C}$. Os modelos matemáticos estabelecidos neste estudo podem ser utilizados para predizer os parâmetros de crescimento das BAL nas condições estudadas (embalagem a vácuo e com ATM) e em condições que não extrapolem a temperatura no intervalo entre $1{ }^{\circ} \mathrm{C}$ e $20^{\circ} \mathrm{C}$. 


\section{ABSTRACT \\ ESTIMATING SHELF LIFE AND GROWTH PARAMETERS OF LACTIC ACID BACTERIA IN COLD CHICKEN BREAST FILLETS PACKED UNDER VACUUM AND MODIFIED ATMOSPHERE}

Lactic acid bacteria (LAB) are part of the natural microflora and correspond to the main deteriorating population of meat products packed under vacuum and modified atmosphere (MAP), and its growth is one factor that defines the shelf life of the product. The aim of this study was to quantify shelf life and growth parameters (lag phase and maximum specific growth rate) of LAB in cold chicken breast fillets packed under vacuum and MAP $(50 \% \mathrm{CO} 2$ and $50 \%$ N2) under different temperatures (1, 4, 8, 12 and $20^{\circ} \mathrm{C}$ ). The growth parameters were estimated with the modified Gompertz, Baranyi and Logistic models, in which the modified Gompertz and Baranyi models presented fits with better statistical indexes (R2, MSE, and bias and accuracy factors), and the Baranyi model showed slightly better fits. The Exponential and Logarithmic secondary models were used to describe the dependence of the parameters with the temperature because showed better statistical indexes. The chicken breast fillets stored with MAP had higher shelf life comparing with vacuum chicken breast fillet. The mathematical models established in this study can be used to predict the growth of LAB packed under vacuum and MAP in the temperature range between 1 and $20^{\circ} \mathrm{C}$.

KEY-WORDS: CHICKEN; PREDICTIVE MICROBIOLOGY; FOOD PRESERVATION.

\section{REFERÊNCIAS}

1 BARANYI, J., ROBERTS, T.A. A dynamic approach to predicting bacterial growth in food. International Journal of Food Microbiology, v.23, p.277-294, 1994.

2 BORCH E, KANT-MUERMANS ML, BLIXT Y. Bacterial spoilage of meat and cured meat products. International Journal of Food Microbiology 33: 103-120, 1996.

3 CAYRÉ ME, VIGNOLO G, GARRO O. Modeling lactic acid bacteria growth in vacuum-packaged cooked meat emulsion stored at three temperatures. Food Microbiology 20: 561-566, 2003.

4 CORTEZ-VEGA WR, PIZATO S, PRENTICE C. Quality of raw chicken breast stored at $5{ }^{\circ} \mathrm{C}$ and packaged under different modified atmospheres. Journal of Food Safety 32: 360-368, 2012.

5 DEGIRMENCIOGLU N, ESNER OK, IRKIN R, DEGIRMENCIOGLU A. Effects of Vacuum and Modified Atmosphere packaging on shelf life extention of minced meat chemical and microbiological changes. Journal of animal and Veterinary Advances 11: 898-911, 2012.

6 FORSYTHE, S.J. Microbiologia da segurança alimentar. Artmed, 2002.

7 GILL AO, GILL CO. Preservative packaging for fresh meats, poultry and fin fish. In: Innovations in Food Packaging. J.H. Han (ed). Elsevier Ltd., New York, p. 204-226, 2005.

8 HOLLEY RA, PEIRSON MD, LAM J, TAN KB. Microbial profiles of commercial, vacuum packaged, fresh pork of normal or short storage life. International Journal of Food Microbiology, 97: 53-62, 2004.

9 IRKIN R, ESMER OK, DEGIRMENCIOGLU N, DEGIRMENCIOGLU, A. Influence of packaging conditions on some microbial properties of minced beef meat at $4{ }^{\circ} \mathrm{C}$ storage. Bulgarian Journal of Agricultural Science 17: 655-663, 2011.

10 JAYAS DS, JEYAMKONDAN S. Modified Atmosphere Storage of Grains Meats Fruits and Vegetables. Biosystems Engineering 82: 235-251, 2002.

11 LATOU, E.; MEXIS, S.F.; BADEKA, A.V.; KONTAKOS S.; KONTOMINAS M.G. Combined effect of chitosan and modified atmosphere packaging for shelf life extension of chicken breast fillets. LWT - Food Science and Technology. V. 55, p. 263268. 2014.

12 KOUTSOUMANIS KP, STAMATIOU AP, DROSINOS EH, NYCHAS GJE. Control of spoilage microorganisms in minced pork by a self-developed modified atmosphere induced by the respiratory activity of meat microflora. Food Microbiology 25: 915-921, 2008. 
13 LONGHI DA, DALCANTON F, ARAGÃO GMF, CARCIOFI BAM, LAURINDO JB. Assessing the prediction ability of different mathematical models for the growth of Lactobacillus plantarum under non-isothermal conditions. Journal of Theoretical Biology 335: 88-96, 2013.

14 NAKASHIMA SMK, ANDRÉ CDS, FRANCO BDGM Aspectos básicos da microbiologia preditiva (Revisão). Brazilian Journal of Food Technology 3:41-51, 2000.

15 NYCHAS GJ, SKANDAMIS PN, TASSOU CC, KOUTSOUMANIS KP. Meat spoilage during distribution. Meat Science 78: 77-89, 2008.

16 RAMIREZ RJC, ULLOA PR, VELAZQUEZ G, ULLOA JA, ROMERO FA. Bactérias Lácticas: Importancia en alimentos y sus efectos en la salud. Revista Fuente 7: 1-16, 2011.

17 RATKOWSKY D, OLLEY J, MCMEEKIN T, BALL A. Relationship between temperature and growth-rate of bacterial cultures. Journal of Bacteriology 149: 1-5, 1982.

18 ROSS, T. Indices for performance evaluation of predictive models in food microbiology. Journal of Applied Bacteriology 81: 501-508, 1996

19 SAMELIS J, KAKOURI A, REMENTZIS J. Selective effect of the product type and the packaging conditions on the species of lactic acid bacteria dominating the spoilage microbial association of cooked of meats at 4 degrees $\mathrm{C}$. Food Microbiology 17: 329-340, 2000.

20 SINGH, R.K.; SINGH, N. Quality of packaged. Foods. Innovations in Food Packaging, ISBN: 0-12-311632-5, p. 24-40, 2005

21 WHITING RC, BUCHANAN RL. A classification of models in predictive microbiology - a reply to K. R. Davey. Food Microbiology 10: 175-177, 1993.

22 ZWIETERING M, JONGENBURGER I, ROMBOUTS F, VANT RIET K. Modeling of the bacterial-growth curve. Applied and Environmental Microbiology 56: 1875-1881, 1990. 